\title{
Automated measure of conditioned orienting behavior in rats
}

\author{
Christopher S. KeEne AND DaVID J. BucCi \\ Dartmouth College, Hanover, New Hampshire
}

\begin{abstract}
The behavioral and neural mechanisms of orienting behavior have interested experimental psychologists for the last several decades. For example, in the framework of associative learning, examining the brain substrates of orienting behavior has yielded significant insight into the neural basis of attentional function and learning. The present study describes a procedure by which the orienting response to a visual stimulus (rearing on the hind legs) can be monitored automatically, and it validates the procedure by comparing data generated by the automated procedure with data generated by the typical observational procedure. The automated procedure provides an inexpensive means of obtaining immediate, online assessment of rearing behavior during a conditioning session, reduces the possibility of experimenter bias, and significantly reduces the time required to evaluate observational data.
\end{abstract}

The presentation or appearance of a stimulus that is novel or otherwise behaviorally relevant often elicits investigative and approach behavior in animals. This response was described early on by Pavlov (1927) as an "investigatory reflex" and is now more commonly referred to as orienting behavior (Sokolov, 1963). The behavioral and neural mechanisms of orienting behavior have interested experimental psychologists for the last several decades, and indeed, there are now entire books dedicated to the topic (see, e.g., Lang, Simons, \& Balaban, 1997). The orienting response (OR) can take many forms, depending in part on the nature of the ORgenerating stimulus. For example, the OR elicited by a visual stimulus typically includes rearing behavior (Holland, 1977), in which the animal stands on its hind legs with both forepaws off the ground (and is distinct from grooming behavior). In the framework of associative learning, analysis of rearing has been used as an index of the associative strength of the conditioned stimulus (CS) that elicits the response (Holland, 1977, 1979; Ross \& LoLordo, 1986) and as an indicator of the associability of the CS (Swan \& Pearce, 1988; Wilson, Boumphrey, $\&$ Pearce, 1992), reflecting the amount of processing, or attention, associated with the stimulus (Kaye \& Pearce, 1984).

Recent studies have gained significant insight into the neural basis of attentional function and learning by examining the brain substrates of orienting behavior. In particular, the central nucleus of the amygdala, cholinergic basal forebrain, hippocampus, and areas of the cerebral cortex have been identified as having critical and potentially different roles in mediating changes in attention and stimulus processing (Baxter, Holland, \& Gallagher, 1997; Bucci \&
Chess, 2005; Bucci, Holland, \& Gallagher, 1998; Chiba, Bucci, Holland, \& Gallagher, 1995; Gallagher, Graham, \& Holland, 1990; Holland \& Gallagher, 1993a, 1993b). For example, damage to the central nucleus of the amygdala disrupts conditioned orienting behavior to a light or a tone, but does not affect unconditioned orienting or conditioned food cup behavior (Gallagher et al., 1990). Conversely, damage to the hippocampus results in loss of latent inhibition and persistence of the OR (Han, Gallagher, \& Holland, 1995).

To date, analysis of the OR to a visual stimulus has relied on observing videotaped behavior of a conditioning session and recording the occurrence of rearing before, during, or after presentation of the CS. Although this method typically produces reliable data, it is often necessary to conduct the analysis after the conditioning session is over. In addition, this method can be very labor intensive and is potentially vulnerable to experimenter bias. The present study describes a procedure by which rearing behavior can be monitored automatically, and it validates the procedure by comparing data generated by the automated procedure to data generated by the typical observational procedure. The results indicate that the automated procedure can provide immediate, online assessment of orienting behavior during a conditioning session, reduce the possibility of experimenter bias, and significantly reduce the time required to evaluate observational data. Indeed, automatic monitoring of other behaviors, such as conditioned food cup behavior and freezing, has already proven to be a useful and valid tool for measuring conditioned responding (see, e.g., Anagnostaras, Josselyn, Frankland, \& Silva, 2000; Baxter, Holland, \& Gallagher, 1999; Marchand, Luck, \& DiScala, 2003).

D. J. Bucci, david.j.bucci@dartmouth.edu 


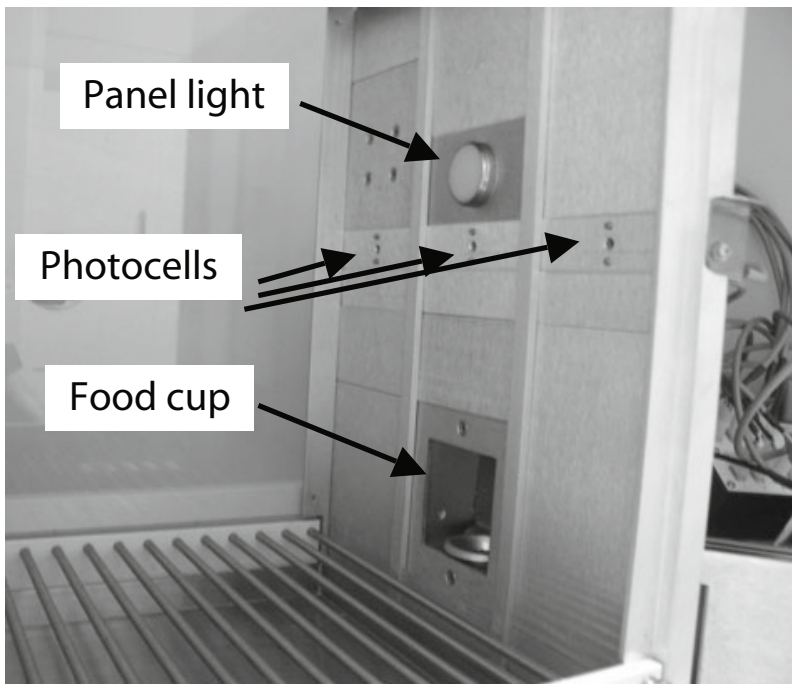

Figure 1. Photograph of the conditioning apparatus, depicting the placement of the stimulus (panel) light, the food cup, and the photocells positioned to detect rearing behavior.

\section{METHOD}

\section{Subjects}

Four male Long-Evans rats ( $325 \mathrm{~g})$ were obtained from Charles River Laboratories (Montreal, Canada). The rats were housed individually and allowed 10 days to acclimate to the vivarium with food available ad lib (Purina standard rat chow). Prior to behavioral training, rats received a craniotomy (sham lesion) in preparation for a subsequent experiment. The rats were anesthetized with isoflurane gas and then secured in a Kopf stereotaxic apparatus (David Kopf Instruments, Tujunga, CA). An incision and retraction of the skin laterally exposed the skull, and burr holes were drilled at the following stereotaxic coordinates: $-2.0,-3.5,-5.0,-6.5,-7.5 \mathrm{~mm}$ from bregma, at $\pm 0.2, \pm 0.2, \pm 0.5, \pm 0.6$, and $\pm 0.4 \mathrm{~mm}$ from the midline, respectively. No injections were made into the holes, and the holes were immediately covered and the wound sutured. After recovering from surgery (14 days), the rats were placed on a restricted food diet and their weights were reduced to $85 \%$ of their ad-lib weights. Throughout the study, the rats were maintained on a 12:12-h light:dark cycle and monitored and cared for in compliance with the Association for Assessment and Accreditation of Laboratory Animal Care guidelines and the Institutional Animal Care and Use Committee.

\section{Behavioral Apparatus}

All components of the behavioral apparatus were obtained commercially from Med Associates Inc., St. Albans, VT. Experiments were conducted using standard operant conditioning chambers $(24 \times 30.5 \times 29 \mathrm{~cm})$ connected to a computer and enclosed in sound-attenuating chambers $(62 \times 56 \times 56 \mathrm{~cm})$ outfitted with an exhaust fan to provide airflow and background noise. The operant chambers consisted of aluminum front and back walls, clear acrylic sides and top, and grid floors. A dimly illuminated food cup (5 $\mathrm{cm}$ in height) was recessed in the center of the front wall, just above the level of the grid floor. A 6-W jeweled panel light, which was the source of the visual CS, was located $11 \mathrm{~cm}$ directly above the top of the food cup.

To detect rearing behavior, three infrared detection units (pairs of photocells) were mounted $15 \mathrm{~cm}$ above the grid floor (see Figure 1). Each unit consisted of a transmitter and receiver, which were mounted opposite each other (i.e., on the front and back walls, respectively). The transmitters were located $1 \mathrm{~cm}$ below the level of the panel light and were evenly spaced on the wall $(8 \mathrm{~cm}$ from the center of one transmitter to the center of the adjacent one). The canister diameter and aperture diameter of the transmitters and receivers were 1.9 and $0.3 \mathrm{~cm}$, respectively, and the transmitters emitted a wavelength of $880 \mathrm{~nm}$. Together, the three beams produced by the photocell units covered the entire area of the chamber, so that a rearing response in any part of the chamber would be detected on at least one photobeam. Because of the distance between the beams and the size of the beam, it was very unlikely that a rear response would be detected by more than one beam.

An additional photocell was placed at the entrance of the food cup to detect head entries into the food cup. All photocells were monitored every $10 \mathrm{msec}$ by the computer. Surveillance cameras located inside each of the surrounding sound-attenuating chambers were connected to a VCR and used to videotape the rats' behavior.

\section{Behavioral Procedures}

Rats were first trained to eat from food cups during a single session in which two 45-mg food pellets (Noyes, New Brunswick, NJ) were delivered at random intervals eight times over the course of a 30-min training session. During each of the subsequent 30-min training sessions (one session per day, for 10 days), the panel light was presented for $10 \mathrm{sec}$ and coterminated with the delivery of two food pellets; this trial was repeated six times during each session on a 5-min variable interval. Rats were weighed and fed at the end of each session to maintain target weights.

\section{Data Collection}

Two primary types of data were collected: computergenerated data and videotape (observational) data. For the computer-generated data, a break in the path of a wallmounted or food-cup-mounted photobeam that lasted at least $50 \mathrm{msec}$ was recorded by the computer. Likewise, reinstatement of the photobeam for a period of $50 \mathrm{msec}$ triggered the computer to stop recording. In this way, the number of breaks in the photobeam was recorded, along with the total amount of time the beam was broken during a designated epoch. Data were collected during four epochs: the 5-sec period preceding presentation of the CS (pre-CS), the first $5 \mathrm{sec}$ of the CS presentation (CS 1), the second $5 \mathrm{sec}$ of CS presentation (CS 2), and the 5sec period after the CS terminated and food was delivered (post-CS). Behavior exhibited during presentation of the 10 -sec CS was separated into two 5-sec periods (i.e., CS 1 and CS 2) because rats typically exhibit mostly rearing behavior during the first half of a 10 -sec presentation of 
A
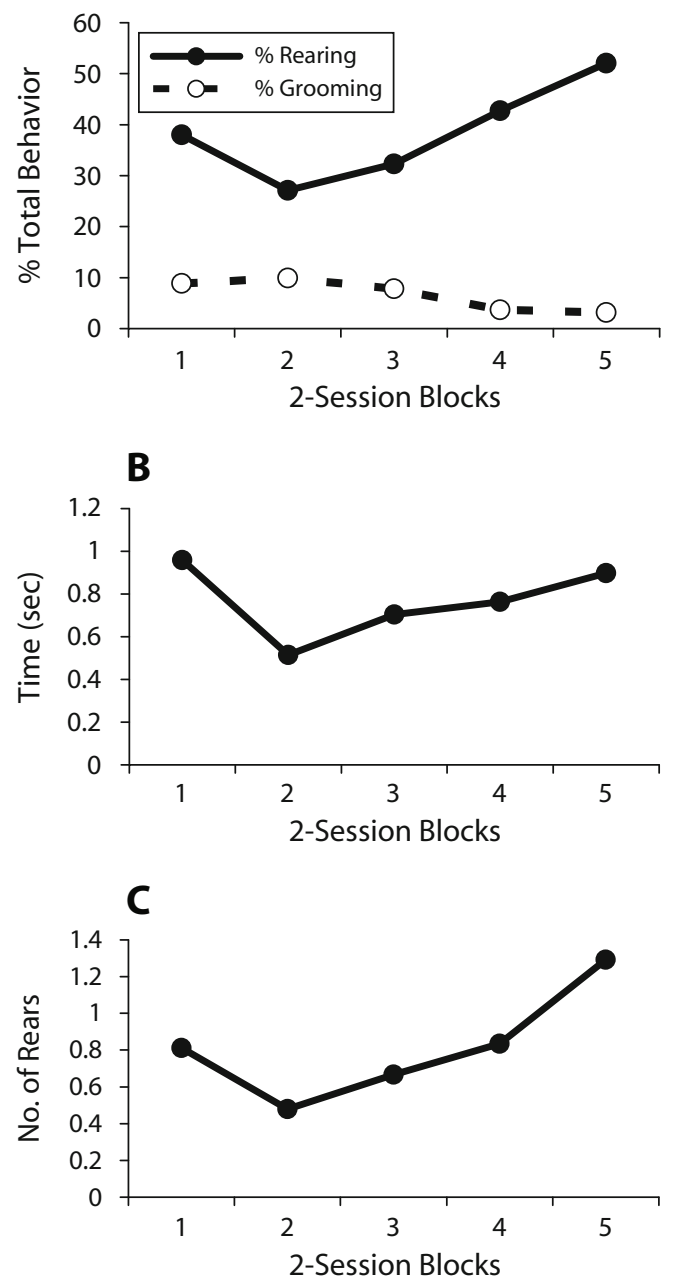

Figure 2. Conditioned orienting behavior (rearing) elicited by presentation of a visual conditioned stimulus. (A) Observations made from videotapes. (B) Time spent rearing. (C) The number of rears. Each of the three measures produced similar patterns of data. Results are averaged across the 4 rats in the study and represent means $\pm S E M$.

a visual CS and mostly food cup behavior during the second half in appetitive conditioning preparations (Holland, 1977). Thus rearing behavior is reported from the first 5 sec of the CS and food cup behavior is reported from the second $5 \mathrm{sec}$ of the CS.

For the observational data, behavior was scored from videotaped recordings of the experiment. Behavior was scored every $1.25 \mathrm{sec}$ during the pre-CS, CS 1 , CS 2, and post-CS periods and recorded as food cup, orienting, grooming, or no behavior. Food cup behavior was defined as nose poke entries into the food cup, head jerk behavior around the food cup, or standing motionless in front of the food cup as described previously (Bucci et al., 1998; Holland, 1984). Orienting to the visual stimulus was defined as standing on the hind legs with both forepaws off the ground (i.e., rearing; Holland, 1977). Grooming was considered to be separate from rearing, even if both forepaws were off the ground. The index of response frequency was percentage of food cup, rearing, or grooming behavior, calculated as the number of times the subject was observed carrying out the target behavior divided by the total observations recorded during an observation period.

\section{Data Analysis}

Data were averaged across the eight trials in each session. Using the session-by-session data, a simple regression was used to assess the relationships among different measures of rearing behavior or food cup behavior. Pearson correlation coefficients were calculated using data pooled from all subjects and statistical significance was assessed using an alpha level of .05. All samples were treated independently regardless of their origin. Additional correlation coefficients were calculated for each subject individually. Paired $t$ tests were used to compare the level of rearing or food cup behavior observed during the presentation of the CS versus behavior observed during the pre-CS period (alpha $=.05$ ).

\section{RESULTS}

\section{Rearing Behavior}

The results obtained with the three different measures of rearing behavior were averaged across the 4 rats and are presented in Figure 2. As shown in panels A-C, similar patterns of rearing behavior were obtained using the observational data (A), the total time the beams were broken (B), and the number of photobeam breaks (C). Indeed, the correlations between each of the measures were statistically significant $\left(r^{2} \mathrm{~s}>.5, p \mathrm{~s}<.0001\right)$. The measures were also significantly correlated in each of the 4 rats when their data were considered individually $(p \mathrm{~s}<.05)$. In contrast, the pattern of rearing behavior (Figure 2A) differed from grooming behavior, and grooming was not significantly correlated with any measure of rearing $\left(r^{2} \mathrm{~s}<\right.$ $.07, p s>.1)$. Furthermore, there was significantly more rearing behavior detected when the CS was presented compared with the pre-CS period according to each of the three measures $(t \mathrm{~s}>6.4, p \mathrm{~s}<.0001)$.

Additional support for the consistency in rearing data provided by each of the measures is shown in Figure 3, which depicts data from an individual animal. Of the 4 rats, this one exhibited the most variability in rearing behavior across sessions, providing an opportunity to determine if each of the three rearing measures captured daily trends in the data. As can be seen in panels A-C of Figure 3 , the patterns of rearing behavior were quite similar when compared across the three measures.

\section{Food Cup Behavior}

The amount of food cup behavior recorded by each method is presented in Figure 4. Similar patterns of food cup behavior were obtained using the observational data, the total time the food cup photobeam was broken, and the number of photobeam breaks, as illustrated in panels A-C. The correlations between each of the measures were statistically significant $\left(r^{2} \mathrm{~s}>.5, p \mathrm{~s}<.0001\right)$. 


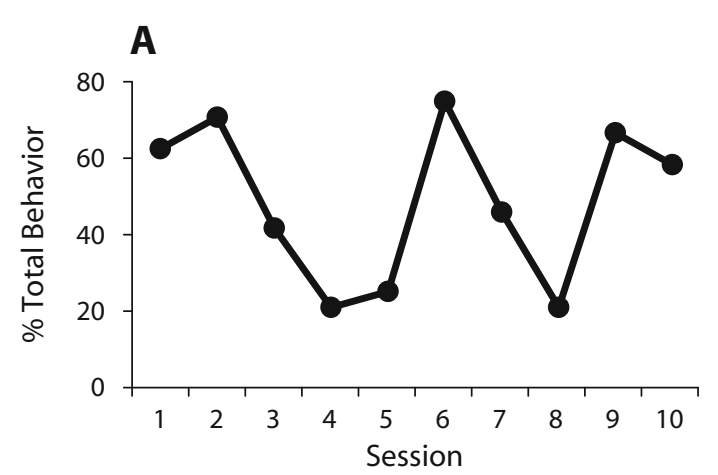

B
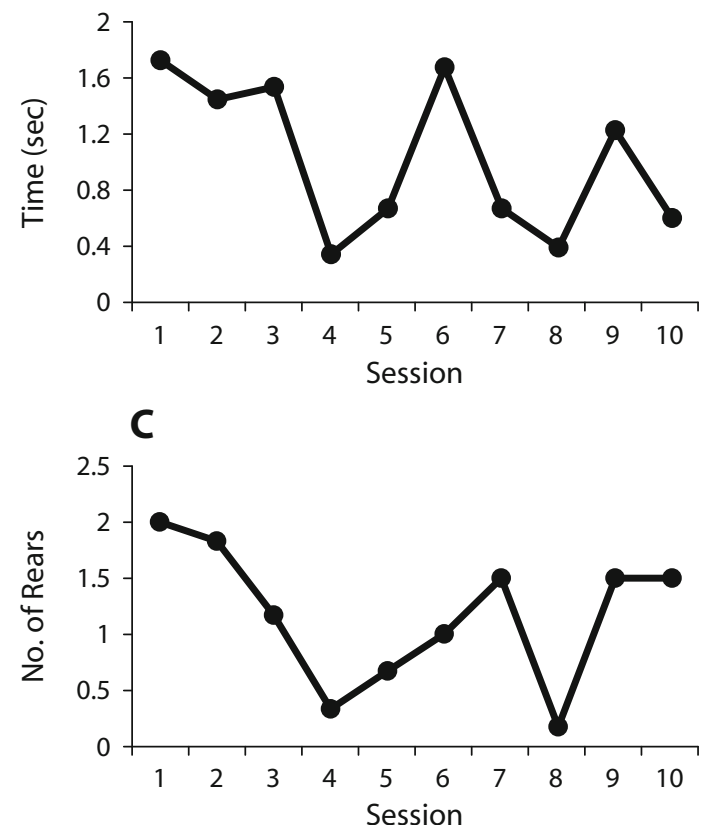

Figure 3. Rearing behavior exhibited by the rat that had the most daily variability in orienting. (A) Observations made from videotapes. (B) Time spent rearing. (C) The number of rears. Each of the three measures produced similar patterns of data.

\section{DISCUSSION}

The present study was designed to validate the use of computer-generated measures of orienting behavior (i.e., rearing) to a visual stimulus in rats. Two types of data were collected by the computer: the number of times an elevated photobeam was broken and the amount of time a beam remained broken when a rat made an orienting response. These measures produced the same pattern of results and were highly correlated with the traditional observational method of assessing rearing behavior. Furthermore, neither of the automated measures was correlated with grooming behavior, and so it appears that the automated measures of rearing are not likely to be confounded by false positives produced when a rat stands on its hind legs and grooms. Last, the rearing data collected by the computer were specific to presentation of the visual stimulus; there was little rearing observed or recorded by the computer during the pre-CS period. These findings indicate that the automated measures of rearing behavior provide a valid and reliable means of evaluating the OR elicited by a visual stimulus.

A comparison between the data generated by the computer and the data obtained through videotape observation reveals a difference in the absolute values of the measures obtained using the two methods. This was not unexpected, and the lower values generated by the computer likely reflect undersampling of rearing and food cup behavior. In the case of rearing, the photocells were purposefully mounted high enough on the wall so that breaks in the beam would not be contaminated by grooming behavior (which typically occurs at a lower height). As a result, it is likely that the computer did not record rearing responses that were relatively low and thus underestimated rearing behavior. Likewise, as described in the Method section, the definition of food cup behavior includes not only nose poke entries
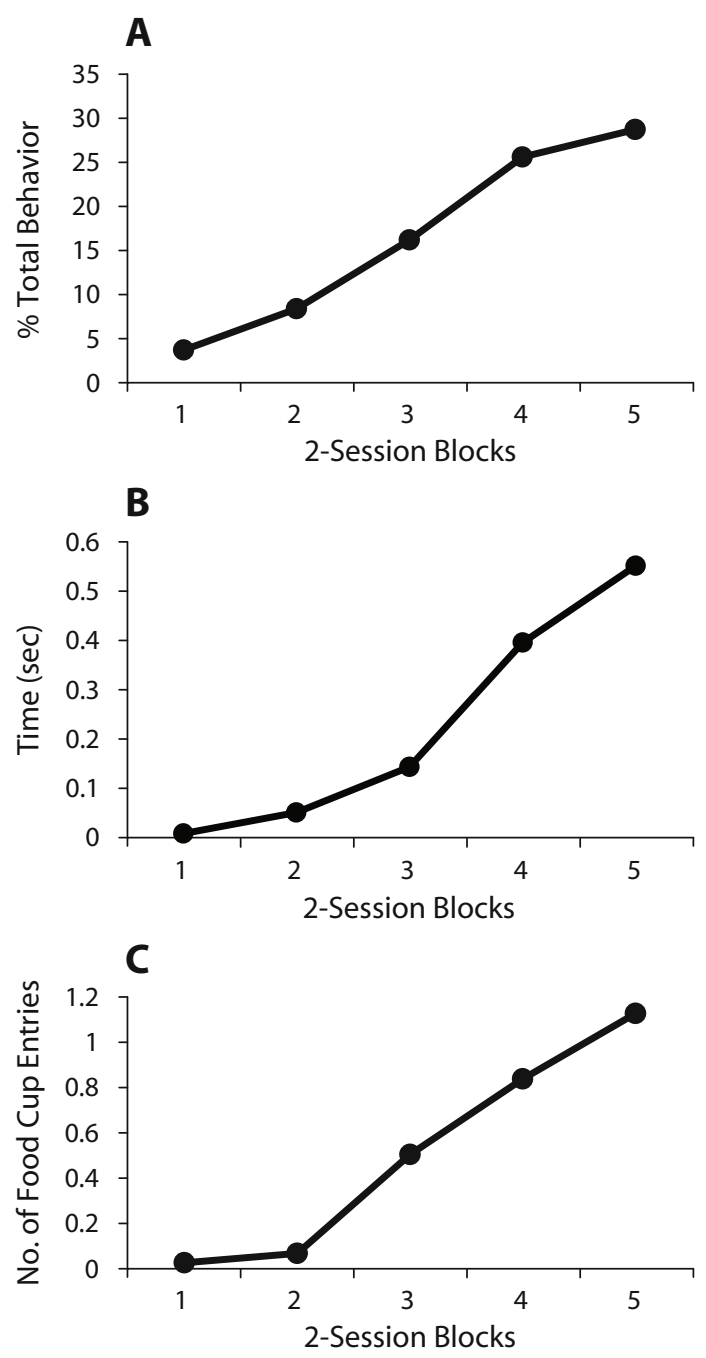

Figure 4. Food cup behavior during presentation of the visual stimulus. (A) Observations made from videotapes. (B) Time spent rearing. (C) The number of rears. Each of the three measures produced similar patterns of data. Results are averaged across the 4 rats in the study and represent means $\pm S E M$. 
into the food cup (which would break the photobeam located just inside the entrance to the cup), but also head jerk behavior around the food cup and standing motionless in front of the food cup (Holland, 1984). By comparison, the computer-generated food cup measures included only nose poke entries into the food cup and thus provided an underestimate. Importantly, however, the patterns of behavior generated by the different methods are all highly similar.

An accurate and valid automated measure of rearing behavior is likely to be very useful, given the substantial number of articles and research focused on the behavioral and neural mechanisms underlying changes in stimulus processing and attention. Indeed, some contemporary learning theories emphasize the importance of changes in the associative strength of CSs in modulating subsequent learning. For example, Pearce and Hall (1980) maintained that the OR is directly tied to the unexpectedness of the stimulus. Novel stimuli or stimuli that have unexpected outcomes undergo increases in processing, whereas the processing of stimuli that reliably predict specific outcomes typically decreases over time. Thus, the processing of CSs undergoes reliable changes that can be assessed by the strength of the OR. Particularly in recent years, the neural processes that regulate these changes in stimulus processing and orienting behavior have become an active area of investigation (reviewed by Holland, 1997).

It is important to note that the present findings were contingent upon accurate and suitable placement of the elevated photobeams designed to capture rearing behavior. The position of the beams is clearly related to the size of the animal, and use of this method requires empirical validation in each laboratory. Likewise, the apparatus described here was designed to record rearing behavior that occurred anywhere in the conditioning chamber. Adjustments could easily be made to record rearing occurring only in certain parts of the chamber-i.e., directly in front of the visual stimulus. This is important because different laboratories have previously defined rearing in response to a visual stimulus to include rearing anywhere in the chamber (Han et al., 1995; Holland, 1979) versus rearing directed particularly to the visual stimulus itself (see, e.g., Kaye \& Pearce, 1984). Adjustments could also easily be made to the height of the photocells depending on how high above the floor the visual stimulus is mounted. In addition, it is noteworthy that the incidence of grooming behavior observed during this type of behavioral procedure is typically very low. If grooming behavior were more frequent, one option would be to use additional photobeams mounted closer to the floor of the chamber and determine if breaks in those beams (presumably caused by grooming and rearing) correlated with breaks in the beams mounted closer to the level of the stimulus light (presumably caused by rearing only). In our experience, the rat's head is more elevated during rearing compared with its height during grooming, and thus the higher photocell would detect only rearing behavior whereas the lower beam would detect both rearing and grooming.

Computer-generated measures of other conditioned responses, such as measures of food cup behavior (see, e.g., Baxter et al., 1999) and conditioned freezing (An- agnostaras et al., 2000; Marchand et al., 2003), have already become commonly used. However, there has yet to be a direct comparison between the data collected with automated measures and that collected through behavioral observation for conditioned food cup and rearing behavior. The present findings indicate that the data generated by the computerized methods used in this study for measuring both rearing and food cup behavior do indeed correlate very highly with data obtained from videotape observations. In addition, the procedures described here are likely to be simpler to use and more cost effective than alternative methods for assessing rearing behavior, such as automated video methods or strain gauges.

In summary, the computer-generated measures of orienting behavior described here are intended to provide a faster assessment of rearing behavior than can be obtained with videotape observation procedures alone. Indeed, these automated methods provide information about orienting behavior as it happens and enable the investigator to make online decisions regarding warranted changes in conditioning procedures. For example, it is often necessary to confirm that processing is indeed decreased before the experiment proceeds to a subsequent conditioning phase of a latent inhibition paradigm. The use of automated measures also reduces the influence of experimenter bias. In addition, it is conceivable that a measure of the time spent rearing and the actual number of rears may be differentially affected by brain lesions or pharmacological manipulations. The availability of both measures of rearing behavior may provide further insight into the neural mechanisms of changes in stimulus processing. Also, the computer-generated measures use continuous variables, which are more amenable to parametric analysis than data generated from observational methods. Nonetheless, the automated procedures described here have potential limitations, such as underestimating the amount of rearing and food cup behavior. Thus they are not meant to replace, but rather to complement traditional observational methods of evaluating the OR.

\section{AUTHOR NOTE}

The research was supported by NIH Grant MH066941 and NSF Grant IBN 0441934. The authors thank Michael K. Simoni for assistance in collecting the data and Robert Leaton for critical comments on earlier versions of the manuscript. Correspondence concerning this article should be addressed D. J. Bucci, Department of Psychological and Brain Sciences, Dartmouth College, 6207 Moore Hall, Hanover, NH 03755 (e-mail: david.j.bucci@dartmouth.edu).

\section{REFERENCES}

Anagnostaras, S. G., Josselyn, S. A., Frankland, P. W., \& Silva, A. J. (2000). Computer-assisted behavioral assessment of Pavlovian fear conditioning in mice. Learning \& Memory, 7, 58-72.

Baxter, M. G., Holland, P. C., \& Gallagher, M. (1997). Disruption of decrements in conditioned stimulus processing by selective removal of hippocampal cholinergic input. Journal of Neuroscience, 17, 5230-5236.

Baxter, M. G., Holland, P. C., \& Gallagher, M. (1999). Blocking can occur without losses in attention in rats with selective removal of hippocampal cholinergic input. Behavioral Neuroscience, 113, 881-890.

BuccI, D. J., \& Chess, A. C. (2005). Specific changes in conditioned responding following neurotoxic damage to the posterior parietal cortex. Behavioral Neuroscience, 119, 1580-1587. 
Bucci, D. J., Holland, P. C., \& Gallagher, M. (1998). Removal of cholinergic input to rat posterior parietal cortex disrupts incremental processing of conditioned stimuli. Journal of Neuroscience, $\mathbf{1 8}$, 8038-8046.

Chiba, A. A., Bucci, D. J., Holland, P. C., \& Gallagher, M. (1995). Basal forebrain cholinergic lesions disrupt increments but not decrements in conditioned stimulus processing. Journal of Neuroscience, 15, 7315-7322.

Gallagher, M., Graham, P. W., \& Holland, P. C. (1990). The amygdala central nucleus and appetitive Pavlovian conditioning: Lesions impair one class of conditioned behavior. Journal of Neuroscience, 10, 1906-1911.

Han, J. S., Gallagher, M., \& Holland, P. (1995). Hippocampal lesions disrupt decrements but not increments in conditioned stimulus processing. Journal of Neuroscience, 15, 7323-7329.

Holland, P. C. (1977). Conditioned stimulus as a determinant of the form of the Pavlovian conditioned response. Journal of Experimental Psychology: Animal Behavior Processes, 3, 77-104.

Holland, P. C. (1979). Differential effects of omission contingencies on various components of Pavlovian appetitive conditioned responding in rats. Journal of Experimental Psychology: Animal Behavior Processes, 5, 178-193.

Holland, P. C. (1984). Origins of behavior in Pavlovian conditioning. In G. H. Bower (Ed.), The Psychology of learning and motivation (Vol. 18, pp. 129-174). New York: Academic Press.

Holland, P. C. (1997). Brain mechanisms for changes in processing of conditioned stimuli in Pavlovian conditioning: Implications for behavior theory. Animal Learning \& Behavior, 25, 373-399.

Holland, P. C., \& Gallagher, M. (1993a). Amygdala central nucleus lesions disrupt increments, but not decrements, in conditioned stimulus processing. Behavioral Neuroscience, 107, 246-253.

Holland, P. C., \& Gallagher, M. (1993b). Effects of amygdala cen- tral nucleus lesions on blocking and unblocking. Behavioral Neuroscience, 107, 235-245.

Kaye, H., \& Pearce, J. M. (1984). The strength of the orienting response during Pavlovian conditioning. Journal of Experimental Psychology: Animal Behavior Processes, 10, 90-109.

Lang, P. J., Simons, R. F., \& Balaban, M. T. (Eds.). (1997). Attention and orienting: Sensory and motivational processes. Mahwah, NJ: Erlbaum.

Marchand, A. R., Luck, D., \& DiScala, G. (2003). Evaluation of an improved automated analysis of freezing behavior in rats and its use in trace fear conditioning. Journal of Neuroscience Methods, 126, 145-153.

PaVlov, I. P. (1927). Conditioned reflexes. (G. V. Anrep, Trans.). London: Oxford University Press.

Pearce, J. M., \& Hall, G. (1980). A model for Pavlovian learning: Variations in the effectiveness of conditioned but not of unconditioned stimuli. Psychological Review, 47, 532-552.

Ross, R. T., \& LoLordo, V. M. (1986). Rearing in response to a visual conditioned stimulus: An index of associative strength or of associability? Learning \& Motivation, 17, 335-346.

Sokolov, E. N. (1963). Perception and the conditioned reflex. Oxford: Pergamon.

Swan, J. A., \& Pearce, J. M. (1988). The orienting response as an index of stimulus associability in rats. Journal of Experimental Psychology: Animal Behavior Processes, 14, 292-301.

Wilson, P. N., Boumphrey, P., \& Pearce, J. M. (1992). Restorations of the orienting response to a light by a change in its predictive accuracy. Quarterly Journal of Experimental Psychology, 44B, 17-36.

(Manuscript received October 28, 2005; revision accepted for publication February 10, 2006.) 